Reprod. Nutr. Dévelop., 1988, 28 (5), 1347-1356

\title{
Pathologie de l'épididyme et répercussion sur la fonction testiculaire
}

\author{
Monique van de CASSEYE, R. SCHOYSMAN ( ${ }^{*}\left({ }^{1}\right)$
}

Laboratoire d'Anatomie Pathologique, Bruxelles. (*) Institut Médico-Chirurgical, Vilvorde, Belgique.

Summary. Epididymal diseases and repercussions on testicular function.

The epididymis is the target of different infections that interfere first with the physiological capacity of the organ and furthermore with the transit of spermatozoa. Epididymal blocks do interfere with testicular function in about $20 \%$ of the cases. According to the pathological agents epididymal blocks will be located either in the initial segments of the epididymis (tuberculosis) or in the lower segments of the organ (gonococcus) or they can interfere with the total structure (chlamydia). Epididymal blocks can be either complete or incomplete and the transit difficulties create lesions of the tubules due to progressive rupture and secondary fibrosis.

\section{Introduction.}

La pathologie épididymaire est vaste et variée. Dans cette revue ne seront cependant prises en considération que les pathologies auxquelles est confronté le clinicien s'occupant de problèmes de stérilité. La pathologie tumorale et la pathologie congénitale de l'épididyme ne seront pas envisagées. Quelle que soit l'origine du processus pathologique, sa séquence morphologique est toujours la même. Seule sa répercussion sur la fonction du transit de l'organe apparaît histologiquement. C'est évidemment la pathologie infectieuse et inflammatoire qui dans ce cadre domine le tableau. Les pathologistes font la distinction entre les épididymites aiguës, les épididymites chroniques et les épididymites granulomateuses (cf. tabl. 1).

L'épididymite aiguë est histologiquement caractérisée par de l'œdème interstitiel, un afflux de polynucléaires dans la lumière des tubes épididymaires et dans l'interstitium. Le processus s'accompagne d'une congestion vasculaire et parfois d'un exsudat fibreux. Belgique.

(1) A qui toute correspondance doit être adressée: 69, avenue R.-Comhaire, 1080 Brussels, 
TABLEAU 1

Pathologie infectieuse de l'épididyme.

\begin{tabular}{|c|c|c|}
\hline & $\begin{array}{l}\text { Lésions } \\
\text { histopathologiques }\end{array}$ & Germes \\
\hline Epididymites aiguës & $\begin{array}{l}\text { œdème interstitiel } \\
\text { congestion } \\
\text { polynucléaires } \\
\text { exsudat fibrineux }\end{array}$ & $\begin{array}{l}\text { gonocoques } \\
\text { chlamydia } \\
\text { E. coli } \\
\text { pseudomonas }\end{array}$ \\
\hline Epididymites chroniques & $\begin{array}{c}\text { fibrose } \\
\text { lymphocytes } \\
\text { micro-abcès } \\
\text { histiocytes } \\
\text { métaplasie épithéliale }\end{array}$ & $\begin{array}{c}\text { E. coli } \\
\text { streptocoques } \\
\text { staphylocoques } \\
\text { pneumocoques } \\
\text {... }\end{array}$ \\
\hline Epididymites granulomateuses & granulomes & $\begin{array}{l}\text { tuberculose } \\
\text { syphilis } \\
\text { lèpre } \\
\text { mycose } \\
\text { brucellose } \\
\text {... }\end{array}$ \\
\hline
\end{tabular}

A ce moment l'organe est gonflé, œdémateux, turgescent et douloureux. La vaginale est atteinte dans $50 \%$ des cas. Les agents les plus fréquemment responsables sont les chlamydia, l'E. coli, et le gonocoque. Le chlamydia a surtout été observé chez les hommes de moins de 35 ans. La pathologie épididymaire due au chlamydia s'installe le plus souvent de façon sub-clinique. L'E. coli a été plus fréquemment mis en évidence chez les hommes de plus de 35 ans. Le gonocoque atteint essentiellement la partie distale de l'organe et respecte la tête de l'épididyme.

L'épididymite chronique est caractérisée par une infiltration inflammatoire de type lymphocytaire, une fibrose dont l'importance varie selon la durée d'évolution du processus pathologique, des micro-abcès plus ou moins enkystés et des remaniements de l'épithélium des canaux épididymaires (métaplasie, formes syncitiales, noyaux bizarres). Les agents les plus fréquemment mis en cause sont I'E. coli, le streptocoque, le staphylocoque, le pneumocoque, etc... L'épididymite granulomateuse est caractérisée par l'unité pathologique «granulome ». L'agent pathogène est celui de la tuberculose, de la syphilis, de la lèpre, une mycose, une brucellose, etc...

Si la tuberculose occupait une place prépondérante dans l'infection épididymaire il y a quelques années, elle a à l'heure actuelle totalement disparu.

Les voies d'accès pour les différents agents pathogènes sont la voie sanguine, canaliculaire (associée aux infections de la prostate ou des vésicules séminales), lymphatique (associée à une infection de la vessie) ou directe en cas de traumatisme. 
Quelles que soient les éthiopathogénies en cause, l'épididyme impliqué dans un processus de stérilité ne possède en fait que quelques types limités de lésions histologiques. L'importance du bloc provoqué par ces lésions et donc la gravité de l'oligospermie dépend de l'extension de ces lésions et de leur localisation (tabl. 2).

TABLEAU 2

Pathologie épididymaire

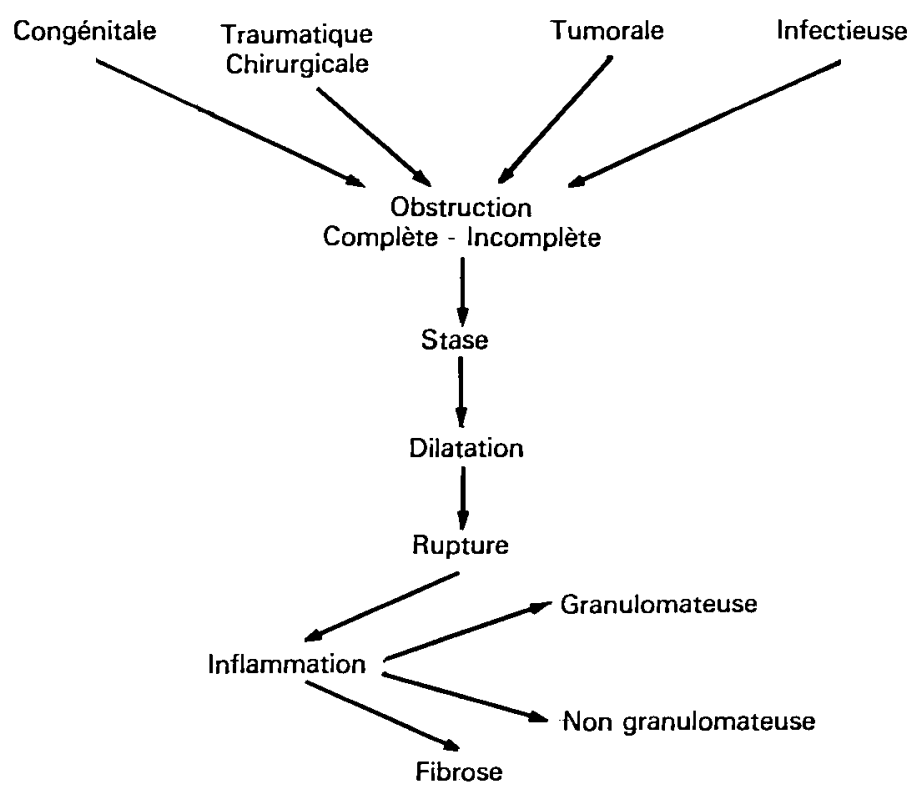

Lésions épididymaires élémentaires (tabl. 3).

TABLEAU 3

Lésions épididymaires élémentaires.

Dilatation kystique - diffuse

- focale

Stase tubulaire

Inflammation interstitielle

Granulomes spermatogéniques

Fibrose interstitielle 
1. Les kystes. - Ils sont localisés ou diffus. Au niveau du kyste on observe une modification de l'épithélium du canal épididymaire. Celui-ci est le plus souvent aplati. Le microscope électronique n'a pas démontré d'altération des jonctions inter-cellulaires. Selon leur localisation et leur importance les kystes entraînent la formation de blocs (fig. 1, 2).

2. La stase. - L'obstruction du canal épididymaire entraîne un arrêt du transit. II en résulte une augmentation de la pression intra-canalaire et une stase de spermatozoïdes en amont de l'obstacle (fig. 3). Dans les zones de stase on a démontré une augmentation de l'activité macrophagique de l'épithélium des canaux (fig. 4). On assiste à une importante accumulation de spermatozoïdes dans la lumière des tubes, les spermatozoïdes entrent en dégénérescence et forment des coagulats basophiles (fig. 5). On peut également observer un afflux de spermiophages dans la lumière des canaux. Les cellules épithéliales se chargent de granules lipofusciniques.

3. Rupture du canal épididymaire. - La rupture du canal entraîne une extravasation de spermatozoïdes au niveau de l'interstitium, ceux-ci suscitent une réaction inflammatoire granulomateuse ou non. Au moment du prélèvement l'infiltrat inflammatoire est le plus souvent discret et de type lymphocytaire. II n'est observé qu'au niveau du tissu fibreux cicatriciel.

4. Granulome. - Le granulome est une formation bien individualisée, essentiellement constituée par une prolifération d'histiocytes. Le granulome est accompagné ou non de cellules géantes. La partie centrale du granulome peut être nécrosée. La prolifération est parfois exubérante et peut atteindre une taille de plusieurs centimètres. Au départ le granulome est centré sur des spermatozoïdes (fig. 6, 7). Petit à petit ceux-ci sont digérés par les histiocytes et laissent en place des acides gras. II se forme à ce moment des granulomes céroïdes (fig. 8). Ces granulomes sont présents dans 2,6\% du matériel autopsique et dans plus de $15 \%$ des cas de vasectomie.

5. Fibrose. - La fibrose remplace progressivement le matériel inflammatoire par une cicatrice modifiant la topographie des tubes, cette fibrose comprend une prolifération de fibres de collagène et de fibres d'élastine.

\section{Répercussion d'un bloc épididymaire sur la fonction testiculaire.}

La comparaison entre une série de 83 biopsies testiculaires faites pendant une intervention pour anastomose et 421 biopsies faites dans des cas de stérilité tout venant, permet d'évaluer les conséquences morphologiques de cet obstacle. Les biopsies testiculaires dans les cas de stérilité ont été pratiquées selon les indications suivantes:

- atteintes testiculaires considérées comme primitives, pas de varicocèle, pas de pathologie de l'axe gonadotrope, pas de pathologie épididymaire opérable ;

- tératospermie, suspicion d'anomalie de la méiose.

Toutes les biopsies ont été évaluées suivant la méthode de Schoysman. Elle prend en compte tous les tubes bien conservés d'un fragment et les répartit en différents groupes selon le degré de maturation de la couche germinative. Elle 


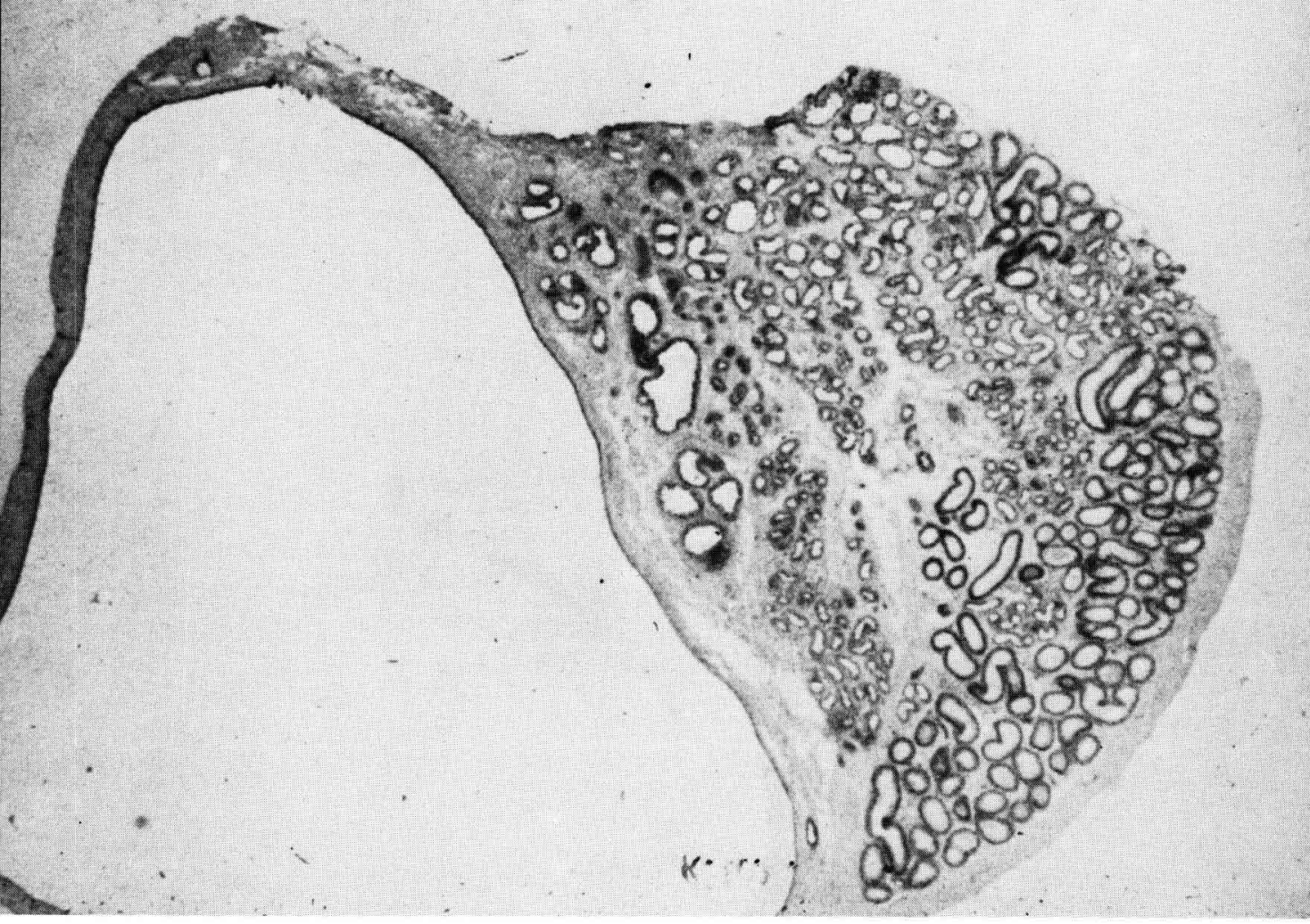

G. 1. - Section de tête épididymaire contenant un kyste.

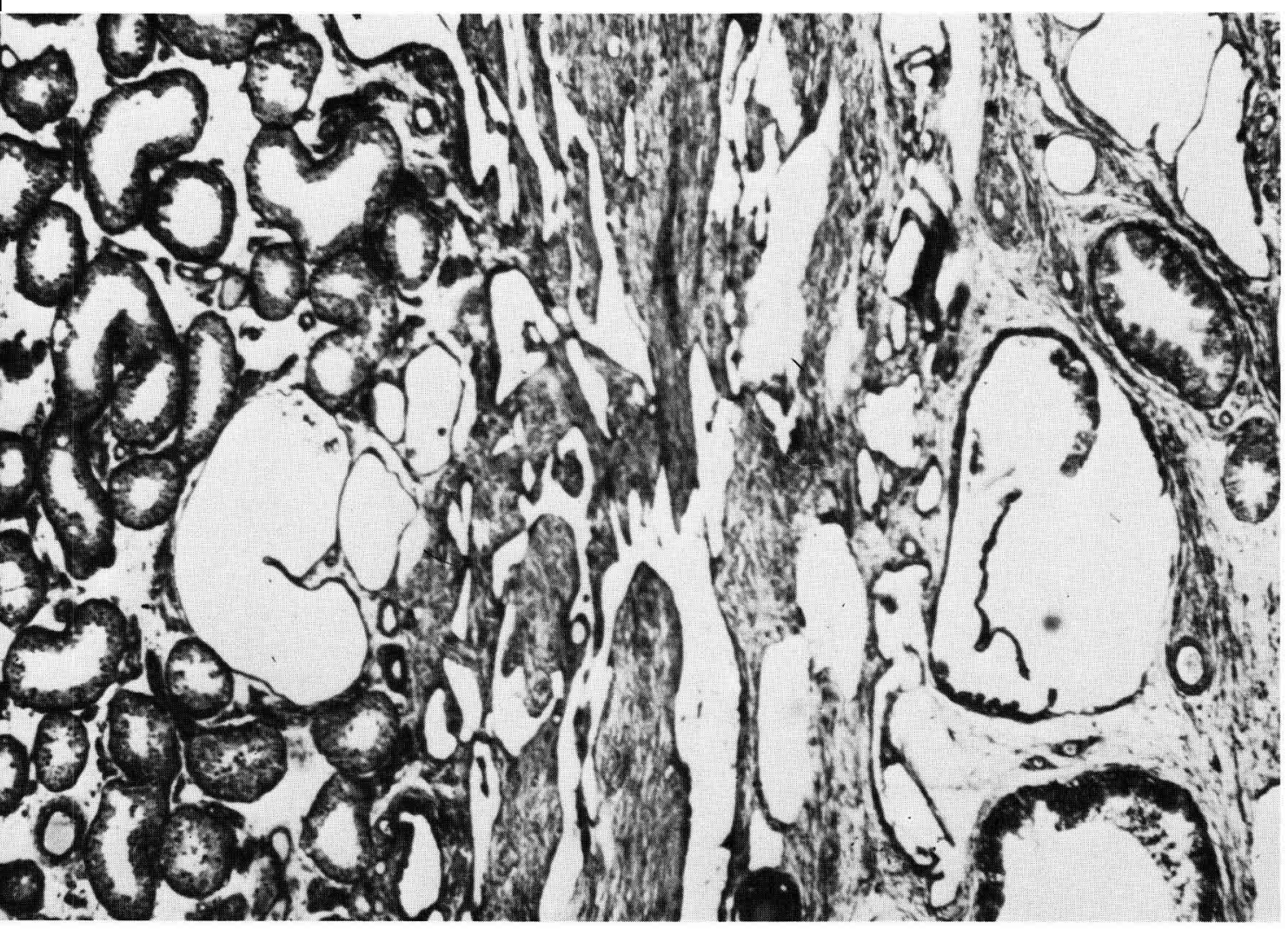

G. 2. - Structures kystiques intra-épididymaires jointes à des structures similaires intra-testiculaires.

Coupe passant par le début de l'épididyme. 


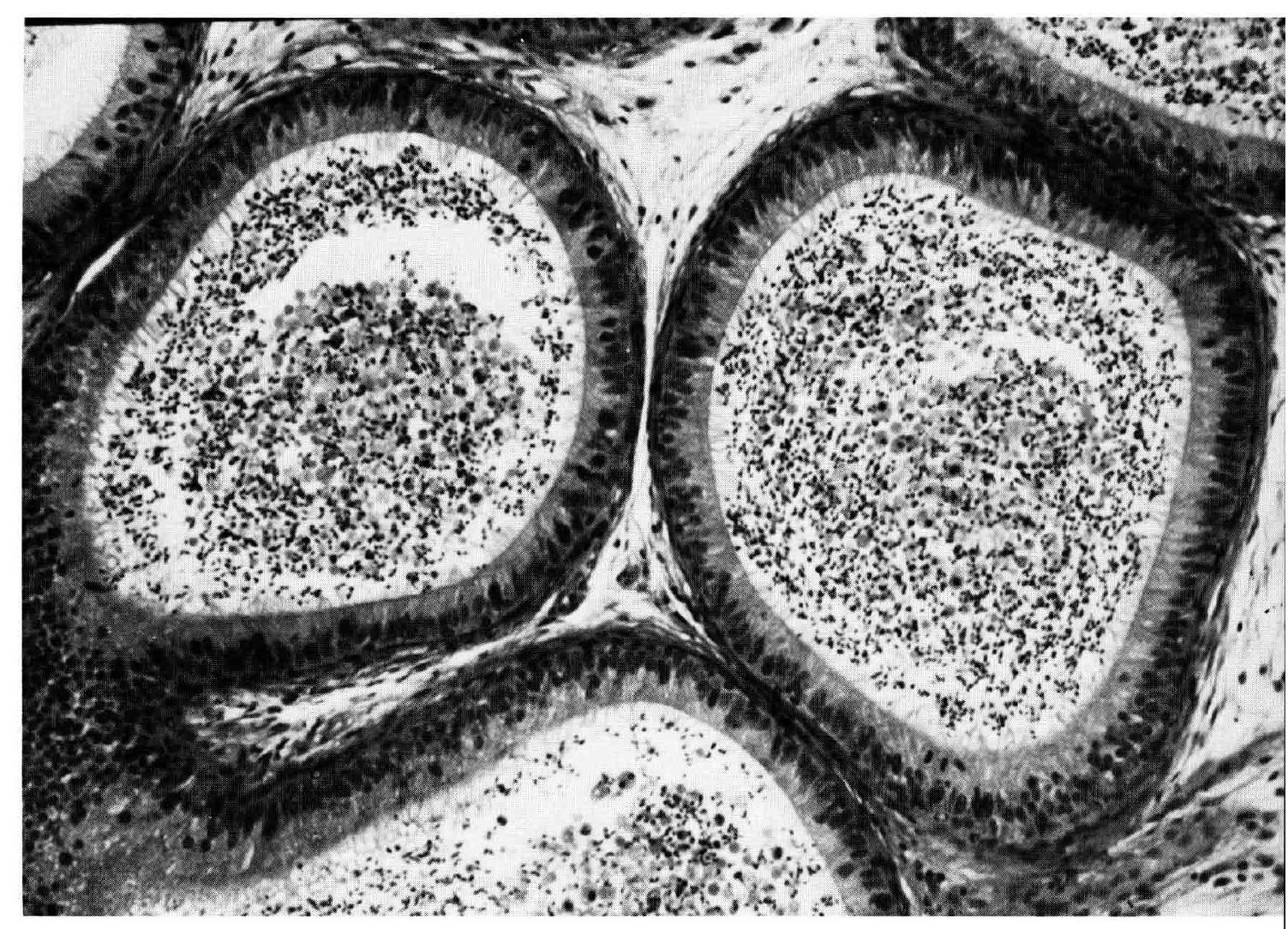

FIG. 3. - Stase spermatique intra-tubulaire en amont d'obstacle épididymaire.

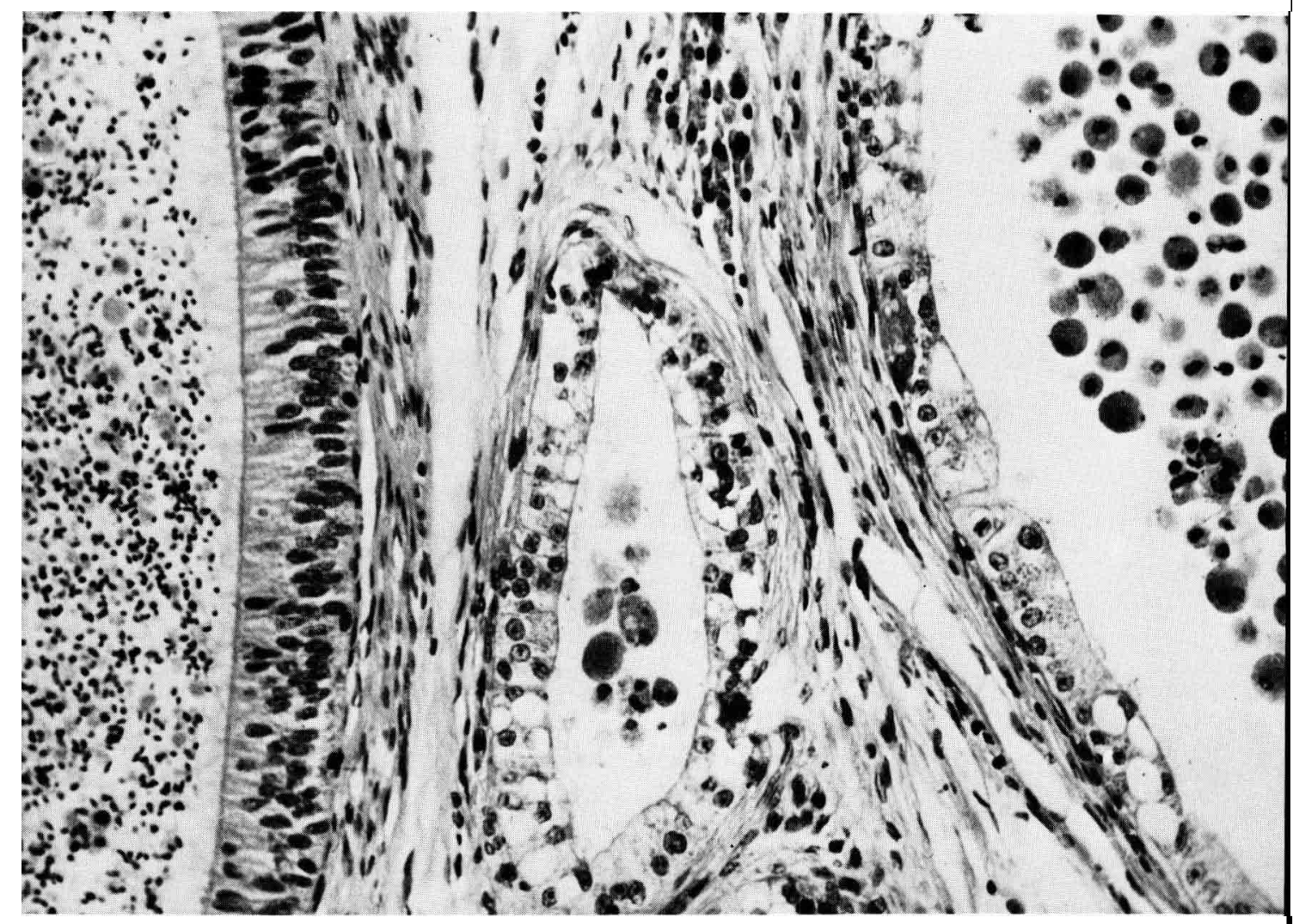


donne une appréciation sur le calibre des tubes et leur paroi, sur l'interstitium et le réseau vasculaire. Si l'on compare les prélèvements faits dans les cas d'obstruction épididymaire (groupe $A$ ) et ceux faits dans les cas de stérilité tout venant (groupe B), on constate que dans le groupe A $80 \%$ des testicules ne montrent aucun signe d'atrophie. Dans le groupe B $65,6 \%$ ne montrent pas de remaniement histologique majeur. Le groupe $A$ contient néanmoins $20,4 \%$ d'atrophie contre $34,4 \%$ dans le groupe B. Cependant $9,6 \%$ des testicules montrent une atrophie sévère préméiotique dans le groupe $A$ contre $19,2 \%$ dans le groupe $B$. Par ailleurs des remaniements mineurs ont été observés dans $33,5 \%$ dans le groupe B contre $18,1 \%$ dans le groupe A (tabl. 4).

TABLEAU 4

\begin{tabular}{|c|c|c|c|c|c|c|}
\hline \multirow[b]{2}{*}{ Normaux } & \multicolumn{2}{|c|}{$\begin{array}{l}\text { Avec bloc } \\
\text { épididymaire }\end{array}$} & \multicolumn{2}{|c|}{$\begin{array}{l}\text { Mansat. A. } \\
1986\end{array}$} & \multicolumn{2}{|c|}{$\begin{array}{l}\text { Sans bloc } \\
\text { épididymaire }\end{array}$} \\
\hline & 42,2 & & - & & 19,7 & \\
\hline Stase & 19,3 & 79,6 & - & 76,0 & 12,4 & 65,6 \\
\hline Rém. mineurs & 18,1 & & - & & 33,5 & \\
\hline Atrophie I & 8,4 & & - & & 13,1 & \\
\hline Atrophie II & 9,6 & 20,4 & - & 24,0 & 19,2 & 34,4 \\
\hline S.C.O.S. & 2,4 & & - & & 2,1 & \\
\hline
\end{tabular}

\section{Conclusions.}

La mise en évidence d'une pathologie épididymaire ne permet pas de répondre de la capacité fonctionnelle de l'organe et son rôle important dans la maturation des spermatozoïdes. L'histopathologie n'illustre en fait que les altérations de la fonction mécanique de transit. II est probable que les oblitérations s'accompagnent dans une certaine mesure de la perte de la fonction physiologique de l'organe. Ceci explique probablement que des anastomoses réussies, même à des niveaux favorables, ne produisent jamais que des spermatozoïdes immobiles.

Les blocs épididymaires ne semblent pas avoir de répercussion majeure sur la fonction testiculaire puisque $80 \%$ des cas gardent une spermatogenèse normale. II est clair cependant que seule une comparaison avec une série de biopsies testiculaires d'hommes fertiles permettrait d'être tout à fait formel sur ce point.

II n'existe pas de corrélation parfaite entre le bilan hormonal et l'état fonctionnel du testicule puisque dans un peu plus de $2 \%$ des cas, la couche germinative est totalement atrophiée et le taux de F.S.H. est normal.

L'aspect morphologique de la pathologie épididymaire et testiculaire n'est qu'une petite fenêtre sur un vaste ensemble fonctionnel extrêmement compliqué dont seul un abord pluridisciplinaire permettra de faire progresser la compréhension. 
Dans le domaine de l'histopathologie les réactions histochimiques offrent certainement des perspectives d'avenir intéressantes.

$5^{e}$ Congrès de la Société d'Andrologie de langue française, Paris, décembre 1987.

\section{Références}

SCHOYSMAN R., 1975. Epididymal oligospermia. Proc. int. Congr. Fertil. Steril., Miami.

SCHOYSMAN R., 1976. La recherche de difficultés du transit épididymaire dans le diagnostic différentiel des patients oligospermiques. Acta Eur. Fertil., No. 1, March.

SCHOYSMAN R., 1984. Microsurgery in infertility. Cofese, Palermo. 